\title{
Commissioning Results from the newly installed KB Refocusing System for the VLS- PGM Beamline at the Canadian Light Source
}

\author{
Lucia Zuin $^{1, *}$, Dongniu Wang ${ }^{1}$, Chithra Karunakaran ${ }^{1}$ \\ 1. Canadian Light Source, Saskatoon, Canada \\ * Corresponding author, Lucia.Zuin@lightsource.ca
}

The Variable Line Spacing-Plane Grating Monochromator (VLS-PGM) beamline [1, 2] at the Canadian Light Source (CLS) is a low energy undulator beamline covering the energy range 5-250 eV. The beamline has been operational since 2005 . The photon energy range is covered with three sets of spherical mirror and plane grating sets with excellent resolving power of more than 10,000 when the exit slit is translated to the exact focus position.

The monochromator focuses the beam to sub-millimeter size $\left(\sim \mathrm{mm}^{2}\right)$ at the sample positions for both the downstream branches (A and B).

While the original beamline design is ideal for examining bulk samples, it limits the beamline's abilities to look at small samples, and to probe spatial distribution of elements and chemicals in the samples. In addition, the $\sim \mathrm{mm}^{2}$ beam-spot results in a relative low overall flux density, which can restrict the sensitivity of many count-rate dependent measurements. In 2009, the Canadian Foundation for Innovation (CFI) awarded funding to the CLS to upgrade the VLS-PGM beamline with a micro-focusing system.

This upgrade involves the construction and installation of a Kirkpatrick-Baez (KB) refocusing system [3] after the exit slit of the branch B. The new system allows for the re-imaging of the original beam spot so that micrometer scale beam spots can be achieved at the sample position even when the exit slit is translated as required to obtain the optimum resolution. With the new focusing optics, theoretical results show that there will be an order of magnitude improvement in flux density with an optimum beam spot size of $\sim 10$ microns.

Points worth emphasizing are that the space between the last mirror pole and the sample position is constrained to $750 \mathrm{~mm}$ and that the beamline section after the KB system is declining at an angle of 9deg. Both the beamline endstations, the XPS and the new, soon-to-be-completed XAS chamber will be able to take advantages of this microfocusing capability of the KB system.

Microfocusing and the tunable polarization (new EPU planned to be installed in 2019), will advance the beamline to a new level for a large number of applications, from element speciation to circuit failure analysis to grain boundaries of polycrystalline materials to space dust investigations, just to name a few. Microbeam also will allow for spectromicroscopy analysis of materials, especially for differentiation of local structures and their distributions in inhomogeneous materials such as catalysts, electrodes for lithium batteries and biological and environmental specimens, hence elemental or even chemical maps with a spatial resolution of several microns could be realized.

In summary, the beamline upgrade with the KB system will enable VLS-PGM, one of the unique beamlines in the world to conduct spectromicroscopy studies of light elements in the 5-250 eV range. 


\section{References:}

[1] R Reininger et al, Rev. Sci. Instrum., 73 (2002), p.1489

[2] Y Hu et al, Rev. Sci. Instrum., 78 (2007), 083109

[3] P Kirkpatrick et al, J. Opt Soc. Am., 38 (1948), p.766

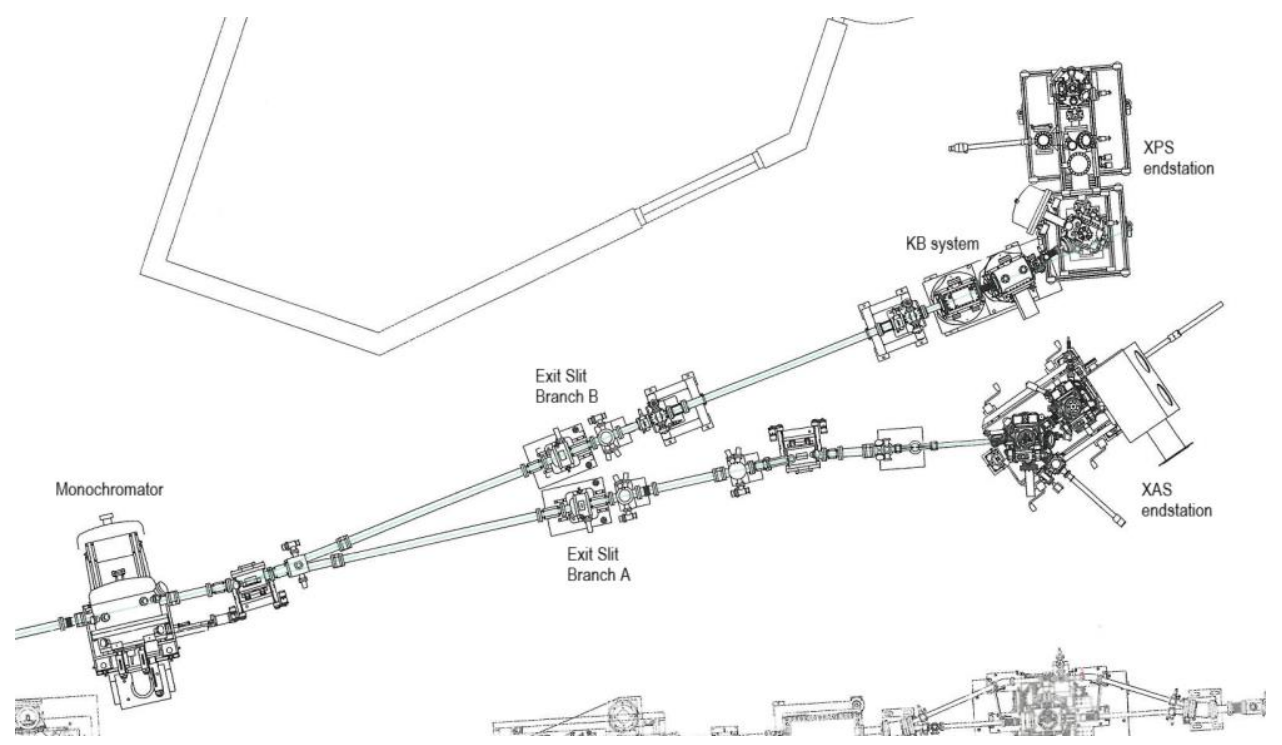

Figure 1. Beamline layout with the schematic of the two new endstations. 